\title{
Perception d'utilité par les patients et les soignants d'un dispositif d'évaluation pédagogique centré sur le développement des compétences des patients : une étude exploratoire
}

\section{Patient and caregiver perception of the usefulness of a pedagogical evaluation system focused on the development of patient competence: exploratory study}

\author{
Claire MARChAND ${ }^{1}$, Jacqueline IGUENANE ${ }^{1}$, Valérie DAVID ${ }^{2}$, Marythé KERBRAT ${ }^{3}$ \\ et Rémi GAGNAYRE ${ }^{1}$ \\ 1 Laboratoire de Pédagogie de la Santé EA 3412, Université Paris 13, Bobigny, France \\ 2 Centre de ressources et de compétences de la mucoviscidose, Pédiatrique, CHU de Nantes, Nantes, France \\ 3 Centre de ressources et de compétences de la mucoviscidose, Roscoff et Réseau Muco Ouest, Roscoff, France
}

Manuscrit reçu le 17 novembre 2009 ; commentaires éditoriaux formulés aux auteurs le 27 février 2010 ; accepté pour publication le 8 mai 2010

\begin{abstract}
Mots clés :
Résumé - Contexte et but : L'évaluation pédagogique constitue une étape importante de Éducation thérapeutique l'éducation thérapeutique du patient (ETP). Cette étude vise à documenter la validité de du patient ; évaluation pédagogique ; compétences ; mucoviscidose : perception d'utilité construit d'un dispositif d'évaluation pédagogique des compétences du patient. Le critère de perception d'utilité du dispositif tant pour les patients que pour les soignants a été retenu pour une première validation. Méthodes : Un questionnaire a été administré à 48 patients atteints de mucoviscidose et ayant participé à trois évaluations à trois mois d'intervalle. Le même type de questionnaire a été proposé aux sept soignants éducateurs ayant réalisé ces évaluations. Résultats : $95 \%$ des patients disent avoir pris plaisir à participer à ces évaluations. Tant du point de vue des patients que des soignants, le dispositif facilite la participation du patient à son évaluation, lui permet de prendre conscience de ses compétences et de discuter avec le soignant des décisions pédagogiques qui en découlent. Parmi les compétences évaluées, celles d'adaptation à la maladie (image de soi, projet de vie, auto efficacité) sont jugées moins souvent utiles pour aider patients et soignants à mieux comprendre comment le patient s'y prend pour gérer sa maladie et pour prendre des décisions adaptées. Ce résultat interroge les programmes d'éducation thérapeutique dans leurs buts et méthodes pour aborder avec les patients leurs compétences d'adaptation à la maladie. Conclusion : Cette étude démontre l'utilité d'un dispositif d'évaluation pédagogique dès lors qu'il respecte des conditions d'utilisation déterminées. Il permet de structurer la rencontre évaluative en prenant en compte un ensemble de compétences et en permettant au patient d'être un véritable partenaire de son évaluation.
\end{abstract}




\section{Keywords:}

Patient therapeutic

education;

pedagogical evaluation;

competencies;

cystic fibrosis;

perception of usefulness
Abstract - Context and purpose: Pedagogical evaluation is an important step of therapeutic patient education (TPE). The study was designed to document the construct validity of a pedagogical evaluation system of patient competencies. The usefulness of the system perception criteria, both for patients and caregivers, was accepted for an initial validation. Methods: 48 patients suffering from cystic fibrosis were given a questionnaire and took part in three evaluations of their competencies staggered over a three-month period. A similar questionnaire was given to the seven caregivers who carried out the pedagogical evaluations. Results: 95\% of the patients appreciated taking part in these evaluations. From both the patient and caregiver viewpoints, the system facilitated the involvement of patients in self evaluation, helped them become aware of their competencies and discuss ensuing pedagogical decisions with caregivers. Among the competencies evaluated, adaptation to the disease (self-image, self-efficacy, life project) was considered less useful in helping patients and caregivers better understand how patients manage their diseases and take appropriate decisions.

The results probed the goals and methods of TEP programs in helping patients develop their disease-management competencies Conclusion: The study demonstrates the usefulness of an educational evaluation system when it adheres to the controlled administration conditions. It helps structure evaluation meetings by taking into account all competencies and allowing patients to act as genuine partners in their evaluation.

\section{Introduction}

Dans le champ de l'éducation thérapeutique du patient (ETP), l'évaluation pédagogique est considérée comme une phase importante ${ }^{[1,2]}$. Par définition, cette évaluation vise à fournir des informations concernant le développement de compétences considérées comme nécessaires pour vivre avec une maladie chronique. Ces compétences sont des compétences d'autosoins ${ }^{[3]}$, des compétences d'adaptation à la maladie ${ }^{[4]}$ et des compétences métacognitives contribuant au développement et au maintien de l'ensemble des compétences du patient. Si des travaux signalent l'importance d'évaluer les compétences du patient ${ }^{[5]}$, nous n'avons pas retrouvé d'études rendant compte d'une évaluation simultanée de l'évolution de plusieurs des compétences qu'un patient doit posséder pour agir sur sa santé. Cela témoigne sans doute de la difficulté de passer d'une évaluation relativement éparse et fragmentée, largement influencée par un paradigme biomédical auquel se référent, même implicitement, les soignants, à une évaluation plus complète ouvrant ainsi un champ de décisions pédagogiques plus pertinentes, comme le sous-tendent des approches systémiques ou holistes ${ }^{[6]}$. À partir de cette hypothèse, nous avons conçu un dispositif d'évaluation pédagogique $^{[7]}$ en nous référant à une approche socio-constructiviste ${ }^{[8]}$. Cette approche considère que l'apprentissage consiste en une construction personnelle de compétences, dépendante des interactions sociales. Pour le patient, ses compétences de soins s'élaborent donc en fonction de ses propres ressources, de son réseau familial, socioprofessionnel et en fonction des interventions d'éducation thérapeutique individuelles et collectives. Le dispositif proposé permet d'apprécier dans un même temps d'évaluation le développement de compétences d'autosoins, d'adaptation à la maladie et les compétences métacognitives des patients ${ }^{[7]}$. Il propose pour l'évaluation de ces compétences des outils et des modalités d'évaluation ainsi que des échelles de mesure pour recueillir les jugements des patients et des soignants. Par ailleurs, les modalités d'utilisation du dispositif d'évaluation sont précisées et sollicitent la réflexion du patient sur l'utilité de ces compétences et sur leur processus de construction. Elles déterminent le caractère formatif de l'évaluation pédagogique proposée. De plus, le dispositif fait appel à un support de visualisation qui permet au patient de se rendre compte de l'état de développement de ses compétences. Ce support favorise selon nous un repérage plus facile de l'ensemble des compétences, qu'un rendu oral ne permet pas toujours. 
Tableau I. Composantes du dispositif d'évaluation pédagogique ${ }^{[5]}$.

\begin{tabular}{|c|c|c|}
\hline $\begin{array}{l}\text { Compétences d'autosoins } \\
\text { et métacognitives }\end{array}$ & Outils/ modalités d'évaluation & Échelles d'évaluation \\
\hline $\begin{array}{l}\text { Comprendre, s'expliquer } \\
\text { Situer son degré de certitude }\end{array}$ & $\begin{array}{l}\text { Questionnaire administré } \\
\text { par le soignant : Test vrai/faux et } \\
\text { degré de certitude }\end{array}$ & $\begin{array}{l}\text { Nombre de connaissances utilisables : justes avec un } \\
\text { degré de certitude élevé ( }>60 \%) \\
\text { Nombre de connaissances dangereuses : erronées avec } \\
\text { un haut degré de certitude }(>60 \%)\end{array}$ \\
\hline \multirow{2}{*}{$\begin{array}{l}\text { Mesurer, analyser, repérer } \\
\text { Décider, faire face }\end{array}$} & $\begin{array}{l}\text { À partir du récit d'un événe- } \\
\text { ment par le patient }\end{array}$ & \\
\hline & $\begin{array}{l}\text { Questionnement d'explicitation : } \\
\text { Qualité de la stratégie de ré- } \\
\text { solution de problème mise en } \\
\text { œuvre par le patient selon des } \\
\text { critères d'appréciation dépendant } \\
\text { du contexte du patient }\end{array}$ & $\begin{array}{l}\text { 1. Inadéquation totale avec conséquences potentielle- } \\
\text { ment dangereuses } \\
\text { 2. Inadéquation avec des conséquences pouvant être } \\
\text { graves à moyen terme } \\
\text { 3. Adéquation favorable la santé du patient et/ou son } \\
\text { entourage } \\
\text { 4. Adéquation efficiente (rapport coût humain, temps } \\
\text { financier, efficacité) favorable à la santé du patient } \\
\text { et/ou son entourage }\end{array}$ \\
\hline $\begin{array}{l}\text { Résoudre un problème thé- } \\
\text { rapeutique, de gestion de sa } \\
\text { vie et de sa maladie, de pré- } \\
\text { vention } \\
\text { Auto-évaluer sa stratégie de } \\
\text { résolution de la situation - } \\
\text { problème } \\
\text { S'adapter à une nouvelle } \\
\text { situation-problème }\end{array}$ & $\begin{array}{l}\text { À partir d'une question d'appel } \\
\text { Capacité d'auto évaluation : Ap- } \\
\text { préciation par le patient de sa } \\
\text { stratégie }\end{array}$ & $\begin{array}{l}\text { 1. Aucune opinion exprimée } \\
\text { 2. Opinion exprimée inappropriée } \\
\text { 3. Opinion appropriée partiellement, guidée } \\
\text { par le soignant } \\
\text { 4. Opinion appropriée et portée spontanément }\end{array}$ \\
\hline Informer son entourage & $\begin{array}{l}\text { Proposition d'une situation } \\
\text { proche et question d'appel: } \\
\text { Capacité d'anticipation adapta- } \\
\text { tion : proposition de straté- } \\
\text { gie dans une nouvelle situation } \\
\text { proche de l'événement décrit }\end{array}$ & $\begin{array}{l}\text { 1. Absence de proposition d'adaptation } \\
\text { 2. Propositions d'adaptation inappropriées } \\
\text { 3. Propositions d'adaptation partiellement appro- } \\
\text { priées insuffisamment justifiée } \\
\text { 4. Propositions d'adaptation appropriées, complètes } \\
\text { et justifiées }\end{array}$ \\
\hline $\begin{array}{l}\text { Pratiquer, faire } \\
\text { identifier les critères } \\
\text { d'efficacité du geste }\end{array}$ & $\begin{array}{l}\text { Observation d'un geste ou d'une } \\
\text { technique à l'aide d'une grille } \\
\text { d'observation } \\
\text { Et/ou }\end{array}$ & $\begin{array}{l}\text { 1. Absence de maîtrise du geste ou de la technique, } \\
\text { danger possible à court terme } \\
\text { 2. Omissions dans le geste ou la technique pouvant } \\
\text { avoir des répercussions à moyen terme } \\
\text { 3. Geste ou technique en cours de maîtrise, favorable } \\
\text { à la santé } \\
\text { 4. Maîtrise du geste ou de la technique, favorable à la } \\
\text { santé }\end{array}$ \\
\hline
\end{tabular}


Tableau I. Suite.

\begin{tabular}{|c|c|c|}
\hline $\begin{array}{l}\text { Compétences d'autosoins } \\
\text { et métacognitives }\end{array}$ & Outils/ modalités d'évaluation & Échelles d'évaluation \\
\hline & $\begin{array}{l}\text { À partir d'une question d'appel: } \\
\text { Identification par le patient des } \\
\text { critères d'efficacité du geste ou } \\
\text { de la technique }\end{array}$ & $\begin{array}{l}\text { 1. Aucun critère d'efficacité n'est cité } \\
\text { 2. Omissions de critères d'efficacité pouvant avoir } \\
\text { des répercussions à moyen terme sur la santé du } \\
\text { patient } \\
\text { 3. Tous les critères d'efficacité sont cités }\end{array}$ \\
\hline \multicolumn{3}{|l|}{$\begin{array}{l}\text { Compétences d'adaptation à } \\
\text { la maladie }\end{array}$} \\
\hline \begin{tabular}{|l|l|} 
Image de soi & \\
\end{tabular} & $\begin{array}{l}\text { A partir d'une question d'appel: } \\
\text { Évaluation de la perception du re- } \\
\text { gard des autres par le patient }\end{array}$ & $\begin{array}{l}\text { Échelle de } 1 \text { à } 10 \\
0 . \text { Regard plutôt négatif } \\
\text { 10. Regard plutôt positif }\end{array}$ \\
\hline Sentiment d'auto efficacité & $\begin{array}{l}\text { À partir d'une question d'appel: } \\
\text { Évaluation de la perception du } \\
\text { patient à pouvoir agir sur des évè- } \\
\text { nements le concernant }\end{array}$ & $\begin{array}{l}\text { Échelle de } 1 \text { à } 10 \\
0 . \text { Je crois que ce que je peux faire n'aura aucune in- } \\
\text { fluence sur mon état de santé } \\
\text { 10. Je crois que ce que je peux faire aura une influence } \\
\text { sur mon état de santé }\end{array}$ \\
\hline $\begin{array}{l}\text { Formalisation d'un pro- } \\
\text { jet/Intention d'engagement }\end{array}$ & $\begin{array}{l}\text { À partir du récit : Évaluation de } \\
\text { la potentialité du patient à envi- } \\
\text { sager un projet à court ou moyen } \\
\text { terme }\end{array}$ & $\begin{array}{l}\text { 1. Impossibilité de formuler un projet } \\
\text { 2. Formulation d'un projet d'ordre général sans envi- } \\
\text { sager de modalités de réalisation } \\
\text { 3. Formulation d'un projet précis, sans modalités de } \\
\text { réalisation } \\
\text { 4. Formulation d'un projet précis avec les modalités } \\
\text { de réalisation }\end{array}$ \\
\hline
\end{tabular}

Le tableau I décrit les composantes du dispositif d'évaluation pédagogique proposé. La figure 1 présente le support utilisé pour visualiser d'une part, les résultats obtenus pour chacune des compétences évaluées et, d'autre part, leur évolution dans le temps.

Le dispositif d'évaluation a été testé auprès de patients atteints de mucoviscidose. Ce choix repose sur le fait que pour vivre avec cette maladie, ces patients doivent mobiliser de nombreuses compétences. Il faut rappeler que la mucoviscidose concerne en France plus de 6000 patients dont près de $40 \%$ sont des adultes avec une espérance de vie de 40 ans. C'est une maladie génétique qui affecte

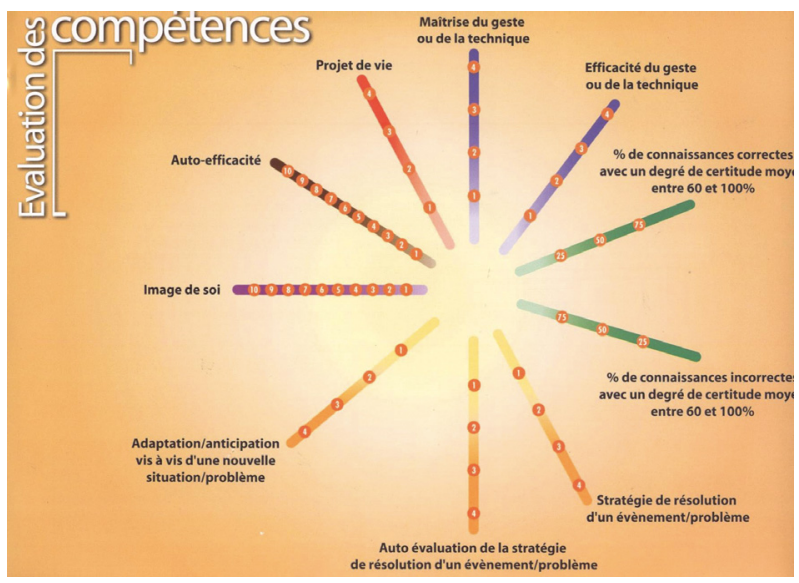

Fig. 1. Support visuel utilisé pour les résultats de l'évaluation pédagogique ${ }^{[5]}$. 
plusieurs organes (poumons, foie, pancréas, tube digestif et organes reproducteurs) et dont le dépistage néonatal est généralisé depuis $2002^{[9]}$. Les patients qui en sont atteints sont, dans leur majorité, suivis dans des Centres de ressources et de compétences de la mucoviscidose $(\mathrm{CRCM})$ répartis sur l'ensemble du territoire français. Ces centres disposent d'une équipe pluridisciplinaire et d'un plateau technique performant. Ils doivent prendre en charge les patients et mettre en œuvre les recommandations nationales validées afin d'apporter une réponse médicale et psychopédagogique appropriée aux problématiques rencontrées par ces patients ${ }^{[10]}$. Dans ces centres, les professionnels ont bénéficié, grâce au soutien de l'association «Vaincre la mucoviscidose », de formations à l'éducation thérapeutique du patient dispensées par l'Institut de perfectionnement en communication et éducation médicales - IPCEM - (organisme de formation en éducation thérapeutique du patient) et respectant les recommandations actuelles de la Haute Autorité de Santé (HAS). Ainsi, les soignants qui prennent en charge les patients atteints de mucoviscidose ont depuis longtemps acquis des compétences d'éducateur, condition importante pour s'approprier le dispositif d'évaluation. De plus, le groupe de travail national en éducation thérapeutique dans le domaine de la mucoviscidose (GETHEM), crée en 2006 et regroupant professionnels de santé et patients, a établi une liste de compétences dont certaines se retrouvent dans le dispositif, ainsi que des conducteurs pédagogiques structurant les activités éducatives et facilitant l'apprentissage des patients ${ }^{[11,12]}$.

\section{Objectifs de l'étude et critère d'utilité}

L'étude présentée dans cet article est de type empirique et exploratoire. L'évaluation pédagogique du patient étant considérée comme un fait établi, nous nous sommes plus particulièrement intéressés à explorer la validité d'un dispositif d'évaluation. Dans un travail précédent ${ }^{[7]}$, nous avons établi que ce dispositif était doté d'une validité de contenu pouvant être qualifiée de relative. En effet, en nous appuyant sur ce qu'énonce la littérature sur les compétences qui doivent être développées par les patients dans le cadre de l'éducation thérapeutique, le dispositif permet d'évaluer ces compétences à partir des choix du patient et partant de situations de sa vie quotidienne, dont la résolution demande de faire appel à un ensemble de savoirs ${ }^{[13]}$. Dans ce contexte, le patient est sollicité à partir de son expérience de prise en charge de ses soins. Ainsi, nous avons fait le choix de documenter la validité de construit de ce dispositif d'évaluation, dans le sens où nous avons cherché à montrer ses éventuelles insuffisances visà-vis de l'évaluation du patient. Pour éclairer cette dimension, nous nous référons au critère de perception d'utilité, entendue comme « (...) la conscience qu'a une personne de la signification ou de la valeur d'un objet perçu et de son habileté à généraliser, à comprendre les implications et à prendre des décisions $(. . .)^{[14]}{ }^{»}$. Pour certains auteurs ${ }^{[15]}$, l'utilité perçue est considérée comme un critère possible pour «faire la preuve, viser l'efficacité tout en conciliant le juste ». Enfin, ce choix est en accord avec une approche socio-constructiviste qui énonce que l'interaction entre deux individus permet le rapprochement ou même la mutualisation de leurs perceptions respectives. Ainsi, c'est par le croisement des perceptions des différents acteurs, l'analyse des similitudes ou des différences, que nous signalerons les limites de ce dispositif d'évaluation.

\section{Méthodes}

L'étude qui s'est déroulée de septembre 2007 à juin 2009 vise à valider un dispositif d'évaluation pédagogique prenant en compte, à l'aide d'un support visuel, différentes compétences d'un patient, qu'elles soient d'autosoins, d'adaptation à la maladie ou métacognitives. Lors de la conception du dispositif, nous avons émis plusieurs hypothèses sur son apport pédagogique pour le patient et le soignant. Celles-ci sont rapportées dans le tableau II. Elles structurent les deux questionnaires utilisés pour cette étude, l'un pour les patients, l'autre pour les soignants.

Le critère d'utilité perçue a été recueilli après trois applications du dispositif auprès des patients 
Tableau II. Hypothèses de l'étude.

\begin{tabular}{|c|c|c|c|c|}
\hline & \multicolumn{2}{|c|}{$\begin{array}{l}\text { 1. Le dispositif d'évaluation influence la } \\
\text { compréhension par le patient et le } \\
\text { soignant de sa (la) situation de santé par } \\
\text { la possibilité }\end{array}$} & \multicolumn{2}{|c|}{$\begin{array}{l}\text { 2. Le dispositif d'évaluation influence } \\
\text { la décision en terme de co-construction, } \\
\text { d'engagement mutuel }\end{array}$} \\
\hline & Pour le patient & Pour le soignant & Pour le patient & Pour le soignant \\
\hline $\begin{array}{l}\text { Les modalités } \\
\text { d'utilisation du } \\
\text { dispositif } \\
\text { d'évaluation } \\
\text { (buts, ordre } \\
\text { d'évaluation des } \\
\text { compétences, } \\
\text { analyse, décision) }\end{array}$ & $\begin{array}{l}\text { 1.1. Mettent en } \\
\text { disposition et } \\
\text { engagent le patient } \\
\text { dans l'activité } \\
\text { d'évaluation et } \\
\text { d'apprentissage }\end{array}$ & $\begin{array}{l}\text { 1.5. Structurent } \\
\text { l'activité } \\
\text { d'évaluation du } \\
\text { soignant dans une } \\
\text { perspective } \\
\text { d'apprentissage du } \\
\text { patient }\end{array}$ & $\begin{array}{l}\text { 2.1. Permettent au } \\
\text { patient de prendre } \\
\text { une position de } \\
\text { refus ou } \\
\text { d'acceptation de } \\
\text { l'évaluation au } \\
\text { départ ou en cours } \\
\text { 2.2. Permettent au } \\
\text { patient de modifier } \\
\text { les modalités de } \\
\text { l'évaluation au } \\
\text { départ ou en cours }\end{array}$ & $\begin{array}{l}\text { 2.5. Permettent au } \\
\text { soignant de vérifier } \\
\text { l'acceptation de } \\
\text { l'évaluation par le } \\
\text { patient } \\
\text { 2.6. Renforcent les } \\
\text { compétences du } \\
\text { soignant de } \\
\text { négociation et } \\
\text { d'aide à une } \\
\text { co-construction }\end{array}$ \\
\hline $\begin{array}{l}\text { Les compétences } \\
\text { et les critères du } \\
\text { dispositif }\end{array}$ & $\begin{array}{l}\text { 1.2. Facilitent l'identifi- } \\
\text { cation de nouveaux be- } \\
\text { soins en faisant le point } \\
\text { sur ses apprentissages } \\
\text { réalisés ou à réaliser } \\
\text { 1.3. Développent des } \\
\text { capacités réflexives sur } \\
\text { sa santé par la recherche } \\
\text { de compréhension, de } \\
\text { questionnement plus si- } \\
\text { gnificatifs de sa part }\end{array}$ & $\begin{array}{l}\text { 1.6. Facilitent l'identifi- } \\
\text { cation de nouveaux be- } \\
\text { soins en faisant le point } \\
\text { sur les apprentissages } \\
\text { du patient réalisés ou à } \\
\text { réaliser } \\
\text { 1.7. Développent un } \\
\text { mode de questionne- } \\
\text { ment chez le soignant } \\
\text { suscitant les capacités } \\
\text { réflexives du patient }\end{array}$ & $\begin{array}{l}\text { 2.3. Permettent au } \\
\text { patient de produire } \\
\text { des hypothèses } \\
\text { de choix } \\
\text { et de décisions } \\
\text { concernant des } \\
\text { compétences à } \\
\text { mobiliser }\end{array}$ & $\begin{array}{l}\text { 2.7. Produisent des } \\
\text { hypothèses d'action } \\
\text { éducatives inattendues } \\
\text { ou imprévues }\end{array}$ \\
\hline $\begin{array}{l}\text { La présentation des } \\
\text { résultats par un } \\
\text { support visuel }\end{array}$ & $\begin{array}{l}\text { 1.4. Rend plus } \\
\text { accessible au patient } \\
\text { ses (sa) transformations }\end{array}$ & $\begin{array}{l}\text { 1.8. Rend plus } \\
\text { accessible au soignant } \\
\text { la transformation } \\
\text { du patient } \\
\text { 1.9. Laisse une trace } \\
\text { écrite des } \\
\text { transformations } \\
\text { du patient }\end{array}$ & $\begin{array}{l}\text { 2.4. Renforce les } \\
\text { décisions sur les } \\
\text { compétences } \\
\text { à mobiliser en } \\
\text { permettant de } \\
\text { s'y référer }\end{array}$ & $\begin{array}{l}\text { 2.8. Attribue une } \\
\text { importance } \\
\text { supplémentaire aux } \\
\text { décisions sur les } \\
\text { compétences à } \\
\text { mobiliser }\end{array}$ \\
\hline
\end{tabular}

ainsi qu'auprès des soignants-éducateurs ayant utilisé le dispositif (le terme de soignant-éducateur est employé ici pour désigner tout professionnel de santé investi dans l'éducation thérapeutique des patients).

\section{Terrain et population}

Le dispositif d'évaluation a été utilisé dans deux CRCM accueillant exclusivement des patients atteints de mucoviscidose et ayant développé depuis plusieurs années des programmes d'éducation thérapeutique. Pour participer à cette étude, les professionnels volontaires ont été choisis selon deux critères. Ils devaient posséder une expérience d'au moins deux ans dans le centre afin d'avoir développé des compétences sur la mucoviscidose et avoir été formés en éducation thérapeutique du patient, leur permettant par la suite de mettre en auvre des pratiques réflexives dans ce domaine. Ces critères sont importants dans la mesure où, s'agissant de recueillir leur perception d'utilité, il semblait 
Tableau III. Modalités d'utilisation du dispositif d'évaluation.

- Expliquer au patient le déroulement de l'évaluation :

- Expliquer la finalité de l'évaluation au patient, son intention formative

- Présenter au patient l'ensemble des compétences évaluées à l'aide du support visuel

- Demander au patient s'il est d'accord pour réaliser cette évaluation

- Demander au patient par quoi il souhaite commencer

- Continuer l'évaluation des compétences de manière systématique et complète

- Demander au patient s'il souhaite aborder une autre compétence

- Reporter les résultats sur le support visuel

- Discuter des résultats avec le patient et prendre avec lui des décisions concernant son suivi éducatif

- Recueillir les réactions spontanées du patient lors de la présentation de ses résultats sur le support visuel

- Question pouvant être posée au patient : quels sont les commentaires qui vous viennent en regardant les résultats sur le support visuel

\section{Consigne pour l'évaluateur :}

- Pendant l'évaluation, éviter de faire des commentaires, de porter un jugement, de proposer des corrections même en présence d'erreurs ou de lacunes importantes

- Solliciter tout au long de l'évaluation la réflexion du patient en posant des questions ouvertes, pour l'accompagner lorsqu'il décrit un geste ou une situation problème

- Reformuler et/ou reprendre ce que dit le patient pour l'aider à approfondir sa réflexion

- Respecter la réflexion du patient

nécessaire qu'ils puissent posséder des éléments de comparaison par rapport à leur pratique de soin et d'éducation quotidienne auprès des patients atteints de mucoviscidose. Par ailleurs, les professionnels devaient avoir suivi une formation à l'éducation thérapeutique centrée sur l'amélioration de leur pratique selon les recommandations de l'OMS ${ }^{[16]}$. Sept soignants-éducateurs ont correspondu à ces critères. Côté patient, il a été envisagé d'administrer le dispositif à 50 patients volontaires, âgés de plus de 16 ans, à condition qu'ils acceptent de participer aux trois séances d'évaluation. Cette pratique étant inhabituelle tant pour les patients que pour les soignants, il nous a semblé qu'ils seraient en mesure de se prononcer sur l'utilité du dispositif à condition que leur expérimentation soit suffisante pour émettre un point de vue critique sur cette expérience.

\section{Déroulement de l'étude et technique de recueil}

Chaque soignant-éducateur a reçu une formation complémentaire de cinq jours sur le dispositif d'évaluation pédagogique. Cette formation a permis aux soignants-éducateurs d'adapter le dispositif aux spécificités de la maladie. C'est ainsi qu'ils ont pris connaissance des modalités d'utilisation du dispositif (tableau III), élaboré les outils du dispositif comme le test de connaissances «vrai/faux », la grille d'évaluation des techniques et gestes, et conçu des situations (évènements) à partir desquelles certaines compétences pouvaient être évaluées. Ils se sont entraînés à son utilisation en travaillant particulièrement sur leur stratégie de questionnement d'évaluation. Pour vérifier sa maîtrise du dispositif, 
chaque soignant a testé au moins une fois son utilisation avec un patient.

Les questionnaires ont été élaborés par un groupe de chercheurs ne participant pas directement aux évaluations. Ils se sont appuyés sur les hypothèses émises et ont sollicité la perception d'utilité des répondants (tableau II). Celle-ci a été étayée à partir des éléments suivants : les modalités d'utilisation du dispositif d'évaluation; les compétences et les critères choisis pour l'évaluation et l'utilisation d'un support visuel pour en présenter les résultats.

Les questionnaires ont été testés auprès de collègues (questionnaire soignant) ayant suivi la formation mais qui n'ont pas souhaité participer à l'étude et auprès de quatre patients pour le questionnaire les concernant. Ces tests ont permis des modifications mineures, comme par exemple la simplification des termes accompagnant les échelles à quatre niveaux.

In fine, les questionnaires soignants et patients, construits selon la même structure, sont constitués principalement de questions fermées et de quelques questions ouvertes. Ces dernières permettent de préciser des éléments de contenu en lien avec une réponse positive à une question fermée. Les échelles de mesure utilisées varient en fonction du degré de nuance accordé aux répondants. Certaines questions appellent selon nous des réponses plus affirmées, alors que d'autres explorent de façon plus fine l'expression du ressenti des répondants.

Les deux questionnaires sont organisés de la manière suivante :

- utilité des modalités d'utilisation : quatre ou cinq questions fermées associées à une échelle de Likert à quatre niveaux ;

- utilité des compétences évaluées : deux questions fermées dichotomiques par compétence évaluée (soit 20 questions) et trois questions fermées dichotomiques associées à une question ouverte en cas de réponse positive ;

- utilité du support visuel : six questions fermées (échelle de Likert ou réponse dichotomique), une question préformée à choix multiple et deux ou trois questions associées à une échelle de type «échelle analogique visuelle» pour évaluer des notions plus complexes telles que le plaisir et la motivation.

Cependant, pour identifier de façon plus prégnante les limites de ce dispositif d'évaluation, nous avons décidé de ramener l'ensemble des réponses obtenues avec les échelles de Likert et les échelles analogiques visuelles à deux positions (utilité perçue ou non par exemple).

\section{Résultats}

Parmi les 50 patients initialement prévus, deux n'ont pas pu se rendre disponibles pour réaliser la troisième évaluation; ils ont été retirés de l'étude. Au total, 48 patients ( 30 hommes et 18 femmes) et sept éducateurs-soignants (quatre infirmiers, un cadre de santé et deux médecins) ont participé à l'étude. La moyenne d'âge des patients est de 29 ans (médiane = 24) ; $40 \%$ sont étudiants. Chez tous sauf un, le diagnostic était fait depuis la naissance (ou depuis les premières années de vie). Cent quarante quatre évaluations ont été réalisées, les 48 patients ayant expérimenté chacun trois moments d'évaluation; $96 \%$ des patients disent avoir bénéficié d'information et de conseils sur la maladie avant cette évaluation. Selon les patients, leur participation à des séances d'éducation thérapeutique évolue au fur et à mesure de l'application des dispositifs d'évaluation : elle concerne $54 \%$ des patients lors de la première application, $60 \%$ lors de la seconde et $71 \%$ lors de la troisième.

Les résultats sont présentés ci-après de façon spécifique pour chaque acteur (patient, soignant) et à partir d'une lecture horizontale du tableau d'hypothèses (tableau II), correspondant à chacune des variables indépendantes proposées (modalité d'utilisation, compétences et critère, support visuel). S'agissant d'une étude exploratoire, il n'a pas été envisagé d'attribuer a priori des poids différents à la vérification de chacune de ces hypothèses.

Utilité du dispositif d'évaluation perçue par les patients

Hypothèses 1.1, 2.1 et 2.2 : Les modalités d'utilisation du dispositif d'évaluation engagent le patient 
Tableau IV. Utilité perçue par les patients des modalités d'utilisation du dispositif et de ses bénéfices.

\begin{tabular}{|l|l|l|}
\hline Les modalités d'administration m'ont : & OUI & $\%$ \\
\hline - Permis de mieux comprendre le but du dispositif d'évaluation $(n=48)$ & 48 & $100 \%$ \\
\hline - Donné le choix de refuser l'évaluation $(n=47)$ & 46 & $98 \%$ \\
\hline - Permis de modifier le déroulement de l'évaluation $(n=47)$ & 46 & $98 \%$ \\
\hline - Permis de commencer par les compétences de mon choix $(n=48)$ & 48 & $100 \%$ \\
\hline - Donné envie de réaliser cette évaluation $(n=48)$ & 43 & $90 \%$ \\
\hline Le dispositif d'évaluation m'a permis de : & & $\%$ \\
\hline - Prendre conscience de ce que je sais faire et compris sur ma maladie $(n=48)$ & 44 & $92 \%$ \\
\hline - Faire le point sur ce que j'ai encore besoin d'apprendre $(n=48)$ & 28 & $58 \%$ \\
\hline - Me poser des questions que je n'ai pas l'habitude de me poser $(n=47)$ & 35 & $74 \%$ \\
\hline
\end{tabular}

dans son évaluation et son apprentissage, lui permettent de refuser l'évaluation ou de modifier son déroulement.

D'une manière générale, $95 \%$ des patients disent avoir pris plaisir à être évalués et leur motivation a été constante au cours des trois évaluations pour $98 \%$ d'entre eux. Le temps d'application du dispositif (en moyenne une heure) a été jugé satisfaisant par la majorité des patients. Les modalités d'administration de l'évaluation mises en place ont permis à tous les patients de mieux comprendre le but du dispositif d'évaluation pédagogique et, par contre coup, celui de l'évaluation même. La majorité des patients souligne que cela leur a donné envie de participer à l'évaluation, qu'ils ont eu la possibilité de modifier son déroulement ou encore de la refuser (aucun patient n'a refusé de participer à l'évaluation).

Hypothèses 1.2, 1.3 et 2.3 : Les compétences et critères du dispositif facilitent l'identification de nouveaux besoins en faisant le point sur ses apprentissages réalisés ou à réaliser, développent des capacités réflexives sur sa santé par la recherche de compréhension de questionnement plus significatifs, permettent au patient de produire des hypothèses d'action sur les compétences à mobiliser face aux situations qu'il rencontre

Les patients disent que l'évaluation leur a permis de prendre conscience de leurs connaissances et de se poser des questions inhabituelles. On note que seulement $58 \%$ d'entre eux pensent avoir pu faire le point sur leurs besoins d'apprentissage (tableau IV). Globalement, la majorité des patients estime que l'évaluation de leurs compétences d'auto soins et métacognitives leur a permis de mieux comprendre la façon dont ils s'y prennent pour gérer leur maladie et de prendre des décisions adaptées à leur santé. Concernant les compétences d'adaptation à la maladie, il ressort que l'évaluation de l'image de soi aide seulement $39 \%$ des patients à mieux comprendre la gestion de leur maladie et à prendre des décisions appropriées. D'ailleurs, dans les commentaires, certains patients précisent que, par exemple, l'évaluation de l'image de soi est difficile à réaliser. Il en est de même pour l'évaluation du projet pour $58 \%$ des patients. En revanche, les patients sont plus nombreux à percevoir une utilité à l'évaluation de leur sentiment d'auto efficacité (tableau V).

Hypothèses 1.4 et 2.4 : La présentation des résultats sur un support visuel rend plus accessible au patient ses transformations

Concernant le support visuel sur lequel les résultats de l'évaluation ont été portés, $87 \%$ estiment qu'il leur permet de visualiser l'évolution de leurs compétences d'une application à l'autre. Le support visuel a incité un peu plus de la moitié d'entre eux à exposer aux soignants leur point de vue sur les apprentissages à réaliser $(77 \%)$ et leur a indiqué les compétences à travailler car jugées insuffisantes (62\%). Le support visuel a été remis à $71 \%$ des 
Tableau V. Utilité de l'évaluation de leurs compétences perçue par les patients.

\begin{tabular}{|c|c|c|c|c|}
\hline & \multicolumn{2}{|c|}{$\begin{array}{l}\text { Comprendre la gestion } \\
\text { de ma maladie }\end{array}$} & \multicolumn{2}{|c|}{$\begin{array}{l}\text { Prendre des } \\
\text { décisions appropriées }\end{array}$} \\
\hline & $\begin{array}{l}\text { Nombre } \\
\text { patients }\end{array}$ & $\%$ & $\begin{array}{l}\text { Nombre } \\
\text { patients }\end{array}$ & $\%$ \\
\hline \multicolumn{5}{|l|}{ Compétences d’autosoins } \\
\hline L'évaluation de mes connaissances m'a aidé à $(n=48)$ & 43 & $90 \%$ & 39 & $81 \%$ \\
\hline $\begin{array}{l}\text { L'évaluation de ma capacité à résoudre des problèmes m'a } \\
\text { aidé à }(n=47)\end{array}$ & 38 & $81 \%$ & 41 & $87 \%$ \\
\hline L'évaluation de la qualité du geste m'a aidé à $(n=48)$ & 43 & $90 \%$ & 40 & $85 \%$ \\
\hline \multicolumn{5}{|l|}{ Compétences métacognitives } \\
\hline $\begin{array}{l}\text { L'évaluation de la confiance dans mes connaissances m'a } \\
\text { aidé à }(n=48)\end{array}$ & 35 & $73 \%$ & 35 & $73 \%$ \\
\hline $\begin{array}{l}\text { L'évaluation des critères d'efficacité utilisés pour le geste } \\
\text { m'a aidé à }(n=47)\end{array}$ & 40 & $85 \%$ & 40 & $85 \%$ \\
\hline $\begin{array}{l}\text { L'évaluation de ma capacité d'auto-évaluation m'a aidé à } \\
(n=47)\end{array}$ & 34 & $72 \%$ & 31 & $66 \%$ \\
\hline $\begin{array}{l}\text { L'évaluation de mes capacités d'adaptation et d'anticipation } \\
\text { m'a aidé à }(n=48)\end{array}$ & 42 & $87 \%$ & 41 & $85 \%$ \\
\hline \multicolumn{5}{|l|}{ Compétences d'adaptation à la maladie } \\
\hline L'évaluation de l'image de soi m'a aidé à $(n=46)$ & 18 & $39 \%$ & 18 & $39 \%$ \\
\hline L'évaluation de l'auto-efficacité m'a aidé à $(n=47)$ & 38 & $81 \%$ & 33 & $72 \%$ \\
\hline L'évaluation de la présence du projet m'a aidé à $(n=48)$ & 28 & $58 \%$ & 30 & $64 \%$ \\
\hline
\end{tabular}

patients. Onze patients ont voulu conserver une trace de l'évaluation, cinq souhaitaient pouvoir s'y référer de temps en temps et quatre patients envisageaient d'utiliser le support visuel pour partager les résultats avec leur entourage.

\section{Utilité du dispositif d'évaluation perçue par les soignants-éducateurs}

Hypothèses 1.5, 2.5 et 2.6 : Les modalités d'utilisation du dispositif d'évaluation structurent l'activité d'évaluation du soignant, permettent de vérifier l'acceptation du patient et renforcent leurs compétences à négocier

Tous les soignants-éducateurs (7/7) estiment que l'évaluation est applicable et sa durée satisfaisante. Leur motivation semble s'être maintenue au cours de trois applications. De même, leur perception sur les modalités d'administration de l'évaluation est très positive et ils observent qu'elles structurent la séance d'évaluation tout en leur permettant d'accueillir les propositions du patient sur son déroulement (tableau VI).

Hypothèses 1.6, 1.7 et 2.7 : Les compétences et critères du dispositif permettent au soignant l'identification de nouveaux besoins en faisant le point sur les apprentissages réalisés, ils développent chez le soignant un mode de questionnement suscitant les capacités réflexives du patient, et produisent des hypothèses d'action éducatives inattendues ou imprévues

Tous les soignants (7/7) notent que l'évaluation du patient les a incités à lui poser des questions pour l'aider à penser sa pratique et à l'exprimer (tableau VI). L'évaluation des compétences d'auto soins et métacognitives du patient permet à la majorité des soignants-éducateurs de mieux comprendre le patient dans la gestion de sa santé et 
Tableau VI. Utilité perçue par les soignants-éducateurs des modalités d'utilisation du dispositif et ses bénéfices sur la pratique éducative $(n=7)$.

\begin{tabular}{|c|l|}
\hline Les modalités d'administration du dispositif m'ont permis de & OUI \\
\hline$\circ$ mieux organiser et structurer le déroulement de l'évaluation & 7 \\
$\circ \quad$ laisser le choix au patient de participer ou non à l'évaluation & 7 \\
$\circ \quad$ accueillir les propositions du patient sur le déroulement de la séance & 7 \\
\hline envie de poursuivre l'organisation de séances d'évaluation & 7 \\
\hline Le dispositif d'évaluation m'a & \\
$\circ \quad$ indiqué précisément les apprentissages réalisés par le patient & 7 \\
$\circ$ identifier les poser des questions suscitant la réflexion du patient & 7 \\
\hline
\end{tabular}

d'envisager avec lui des activités d'éducation thérapeutique. Concernant les compétences d'adaptation à la maladie, moins de la moitié des soignantséducateurs (3/7) estime qu'évaluer l'image que le patient a de lui-même (image de soi) les aide à mieux comprendre le patient dans la gestion de sa santé et seulement deux sur sept considèrent que l'évaluation de l'auto efficacité du patient leur permet d'envisager des activités d'éducation thérapeutique avec le patient (tableau VII).

Hypothèses 1.8, 1.9 et 2.8: La présentation des résultats sur un support visuel rend plus accessible au soignant la transformation du patient, laisse une trace écrite et attribue une importance supplémentaire aux décisions sur les compétences à mobiliser

Pour le support visuel (figure 1), les sept soignants-éducateurs estiment qu'il leur permet de visualiser le développement des compétences du patient. Cependant, seuls quatre d'entre eux pensent que cela leur permet de négocier avec le patient les compétences à mobiliser dans la gestion de sa santé. Le support visuel constitue pour quatre soignantséducateurs un outil de référence permettant de faire le lien entre les séances d'éducation, de communiquer en équipe $(5 / 7)$ et de conserver une trace de l'évaluation. Par contre, moins de la moitié des répondants (3/7) pense utiliser ce support pour communiquer avec le médecin traitant du patient.

\section{Discussion}

Les résultats recueillis n'ayant pas permis de pondérer l'influence de chacune des hypothèses sur la perception d'utilité des répondants, la discussion cidessous est organisée de façon à mettre en valeur ce qui pourrait être des avantages du dispositif d'évaluation et ce qui en constitue déjà des limites.

Un dispositif d'évaluation utile pour structurer l'activité d'évaluation pédagogique

Comme en témoignent les résultats, les patients et les soignants-éducateurs estiment que le dispositif d'évaluation, tel qu'il est mis en place, facilite la participation du patient dans cette activité évaluative. En effet, les soignants-éducateurs ont toujours donné la possibilité au patient de refuser ou de modifier le déroulement de l'évaluation (tableaux IV et VI). Cependant, bien que le cadre formatif de l'évaluation soit particulièrement expliqué au patient au début de l'évaluation (possibilité de s'exprimer librement, absence d'enjeu de jugement pendant l'évaluation, etc.), on ne peut pas exclure un phénomène de désirabilité sociale qui influencerait en positif les réponses des patients, en particulier parce que les patients et les soignants se connaissent depuis longtemps. Malgré tout, les résultats obtenus permettent de considérer comme valide une des 
Tableau VII. Utilité perçue par les soignants-éducateurs de l'évaluation des compétences du patient $(n=7)$.

\begin{tabular}{|c|c|c|}
\hline & $\begin{array}{l}\text { Comprendre la gestion de } \\
\text { la maladie par le patient }\end{array}$ & $\begin{array}{l}\text { Envisager des activités } \\
\text { d'ETP adaptée au patient }\end{array}$ \\
\hline & Nombre de soignants & Nombre de soignants \\
\hline \multicolumn{3}{|l|}{ Compétences d'autosoins } \\
\hline L'évaluation des connaissances du patient m'a aidé à & 6 & 7 \\
\hline $\begin{array}{l}\text { L'évaluation de la capacité du patient à résoudre des pro- } \\
\text { blèmes m'a aidé à }\end{array}$ & 7 & 6 \\
\hline L'évaluation de la qualité du geste du patient m’a aidé à & 7 & 7 \\
\hline \multicolumn{3}{|l|}{ Compétences métacognitives } \\
\hline $\begin{array}{l}\text { L'évaluation de la confiance du patient dans ses connaissances } \\
\text { m'a aidé à }\end{array}$ & 6 & 6 \\
\hline $\begin{array}{l}\text { L'évaluation des critères d'efficacité utilisés par le patient } \\
\text { pour évaluer son geste m'a aidé à }\end{array}$ & 7 & 6 \\
\hline $\begin{array}{l}\text { L'évaluation de la capacité d'auto-évaluation du patient m'a } \\
\text { aidé à }\end{array}$ & 7 & 5 \\
\hline $\begin{array}{l}\text { L'évaluation des capacités d'adaptation et d'anticipation du } \\
\text { patient m'ont aidé à }\end{array}$ & 7 & 6 \\
\hline \multicolumn{3}{|l|}{ Compétences d'adaptation à la maladie } \\
\hline $\begin{array}{l}\text { L'évaluation de l'image de soi que le patient a de lui-même } \\
\text { m'a aidé à }\end{array}$ & 3 & 4 \\
\hline L'évaluation de l'auto-efficacité du patient m'a aidé à & 4 & 2 \\
\hline L'évaluation de la présence du projet du patient m'a aidé à & 5 & 5 \\
\hline
\end{tabular}

hypothèses, selon laquelle les conditions de mise en œuvre de l'évaluation influencent l'engagement du patient et obligent le soignant à vérifier systématiquement son acceptation. D'autre part, tous les soignants-éducateurs soulignent que les modalités de mise en œuvre de l'évaluation ont comporté un effet structurant de l'activité d'évaluation tout en leur permettant d'accueillir les propositions du patient. Cela confirme l'idée selon laquelle poser un cadre pédagogique facilite les échanges, la créativité et constitue une aide à la communication ${ }^{[17]}$.

\section{Un dispositif d'évaluation permettant d'objectiver les compétences des patients}

Les avis des patients et des soignants divergent en ce qui concerne la capacité du dispositif d'évaluation à identifier de nouveaux besoins d'apprentissage. En effet, seuls $58 \%$ des patients estiment que l'évaluation leur a permis d'en déduire des besoins d'apprentissage alors que tous les soignants estiment que ce dispositif d'évaluation leur permet d'identifier les besoins d'apprentissage du patient (tableau VI). Ce résultat relativement discordant entre les patients et les soignants peut s'expliquer en partie par la prise de conscience des patients, au moment de l'évaluation, qu'ils possèdent des compétences et un niveau de connaissances élevé (92\% estiment qu'ils ont pris conscience des compétences qu'ils possèdent). Cette prise de conscience primerait chez le patient au moment de l'évaluation et ne lui permettrait pas d'enchaîner sur un autre processus complexe que représente l'analyse de ses besoins d'apprentissage. Une autre explication pourrait être rattachée aux résultats obtenus par les patients à la première application et à leur évolution au cours des deux autres applications. La présente étude n'avait pas pour objectif d'analyser 
spécifiquement ces résultats. Cependant, il est probable que les patients aient, dès la première application, un niveau de connaissance et de compétences relativement élevé compte tenu des séances d'éducation dont ils ont bénéficié depuis longtemps, la plupart d'entre eux étant pris en charge depuis leurs premières années de vie. Si cette évaluation ne révèle pas pour les patients de nouveaux besoins d'apprentissage, elle a néanmoins pour conséquence d'objectiver les compétences qu'ils possèdent, ce qui peut influencer leur sentiment d'efficacité personnelle ${ }^{[18]}$. Ces constats renforcent le principe qu'il est fondamental de proposer au patient des temps d'évaluation formative ${ }^{[13]}$ dont les résultats discutés avec le soignant donnent lieu, selon plus de la moitié des patients, à des prises de décisions pédagogiques adaptées.

Cette procédure permet également au patient de prendre conscience de ce qu'il sait, de réaliser qu'il le sait et donc qu'il peut le mettre en pratique. Ce processus de mentalisation aide le patient à fonder ce qui vaut pour lui et participe à définir sa propre norme de santé, en interaction avec la norme évaluative des soignants. Le développement de cette auto normativité du patient permettrait de soutenir sa motivation à trouver un équilibre et une harmonie satisfaisants entre sa maladie et sa vie quotidienne ${ }^{[19]}$.

Par ailleurs, les conditions de mise en œuvre de l'évaluation ont eu le mérite de mettre le patient en position d'auto-évaluation, ce qui constitue un apprentissage métacognitif important ${ }^{[20]}$ favorisant en retour, selon certains auteurs ${ }^{[21]}$, la mise œuvre des compétences d'autosoins.

Un dispositif d'évaluation qui interroge l'utilité perçue des compétences d'adaptation à la maladie

Les résultats obtenus montrent que l'évaluation des compétences d'autosoins et métacognitives est perçue comme utile, autant pour les patients que pour les soignants. Ce temps d'évaluation aide les soignants-éducateurs à mieux comprendre comment le patient s'y prend pour gérer sa maladie et pour prendre des décisions adaptées à sa situation de santé (tableaux V et VII). Dans cette étude, l'évaluation des compétences d'adaptation à la maladie s'est limitée à trois processus : l'image de soi, le sentiment d'auto efficacité, le projet. Or, l'évaluation de l'image que le patient a de lui-même (compétence d'adaptation à la maladie) est moins souvent perçue comme utile puisque seuls $39 \%$ des patients lui reconnaissent une utilité pour mieux comprendre la gestion de leur maladie et prendre des décisions appropriées pour leur santé. D'ailleurs trois soignantséducateurs partagent ce point de vue. S'il est reconnu que l'image que le patient a de lui-même influence son comportement de santé ${ }^{[22]}$, le lien, tant pour le patient lui-même que pour le soignant, ne semble pas évident. Par ailleurs, les soignantséducateurs constatent au cours des évaluations que la question sur l'image de soi reste difficile à recevoir pour le patient et délicate à poser de leur part, même si elle est indirecte comme c'est le cas dans cette évaluation. Ainsi pour éviter une question trop directe sur l'image de soi, qui pourrait heurter la sensibilité des patients, nous avons choisi de poser une question qui demande au patient comment il perçoit le regard des autres sur lui ${ }^{[7,23]}$. Cependant, questionner le patient sur la perception qu'il a de lui-même et lui demander de réfléchir sur comment il se juge et s'estime peut déclencher une émotion importante, sans doute inhabituelle, à l'origine de cette difficulté cognitive à reconnaître son intérêt. Le choix de cette question peut être discuté dans son éclairage encore trop brutal, même si tous les patients sauf un se sont prononcés sur leur «image de soi ». Il est donc possible que l'évaluation de l'image de soi soit difficile à réaliser pour le patient qui ne saisit pas l'influence que cet état peut avoir sur la façon dont il gère sa maladie et prend des décisions relatives à sa santé. Par ailleurs, il est probable que l'image de soi, comme le sentiment d'efficacité, soient variables en fonction des situations rencontrées par le patient. Ceci nous conduit à envisager des améliorations au niveau du dispositif d'évaluation pédagogique, en proposant d'évaluer ces compétences d'adaptation à la maladie en lien direct avec une compétence d'autosoins et des 
situations rencontrées par le patient. Du côté des soignants-éducateurs, il est possible que cette question sur «l'image de soi » soit jugée très personnelle, intrusive, d'où probablement une difficulté à la poser et se convaincre de son utilité dans l'ETP. De même, concernant l'évaluation du projet, seulement la moitié des patients pense que son identification les aide à mieux comprendre leur maladie et, pour un peu plus de la moitié, qu'elle influence leurs décisions concernant leur santé. Une hypothèse est que les patients interrogés, malades depuis leur naissance, ont régulièrement eu à élaborer des projets puis à les modifier en fonction de l'évolution de leur santé. Cependant, la question du projet reste cruciale car il est décrit que la réalisation d'un projet dépend de l'état de santé du patient et de sa capacité à prendre des décisions appropriées à sa situation ${ }^{[19]}$.

Enfin, on peut noter que, dans le dispositif, ces notions (l'image de soi et le projet) sont les seules à ne pas être formulées comme une compétence. Leur libellé ne renvoie pas suffisamment le patient à une activité concrète dans la vie de tous les jours. Il serait peut-être nécessaire de formuler des compétences dont la mobilisation est intimement liée à l'expérience et au vécu du patient.

De manière générale, ce constat souligne la nécessité pour les soignants et les patients de travailler davantage dans les programmes d'éducation thérapeutique sur la relation pouvant exister entre des compétences d'adaptation à la maladie et celles d'autosoins. Les expériences créatives sollicitées chez les patients, par exemple, prennent ici toute leur place ${ }^{[24]}$. En effet, il est difficile de tout faire reposer sur la situation d'évaluation et d'attendre qu'elle révèle des apprentissages qui n'auraient été que peu abordés dans le programme d'éducation thérapeutique ou dans la vie quotidienne du patient.

\section{Un dispositif d'évaluation qui nécessite des conditions d'application}

Il ressort de cette étude que le dispositif tel qu'il a été construit peut être diffusé et utilisé par les soignants, à condition que ceux-ci élaborent en équipe des outils d'évaluation valides, et déterminent des critères d'évaluation pour chacune des compétences à évaluer. Cependant, certains patients ont émis des commentaires selon lesquels la troisième application entraînait un effet de lassitude. Ils proposent de varier les outils (notamment le questionnaire d'évaluation des connaissances et les critères et modalités d'évaluation des compétences métacognitives). Il est possible que ce sentiment soit lié au fait que, pour les besoins de l'étude, les applications étaient trop rapprochées dans le temps (une application tous les trois mois environ). Néanmoins, cette remarque souligne l'importance de développer une réflexion régulière autour de l'évaluation afin d'en améliorer les situations, les procédures et les outils. Celle-ci nécessite d'être planifiée à l'avance mais doit aussi, pour une plus grande contextualisation, faire l'objet d'une adaptation (en particulier pour les situations d'évaluation) au moment même de son administration. Il est indiscutable qu'un compromis est à définir entre la recherche d'une validité de construction et l'authenticité des situations d'évaluation ${ }^{[13]}$.

Dans cette étude, les patients et les soignants estiment la durée d'administration de l'évaluation acceptable. Il ne faut pas oublier que cette étude prend place dans des centres spécialisés qui ont fondé une organisation de prise en charge adaptée à la situation des patients atteints de mucoviscidose. Cependant, il n'est pas fait mention d'une limite en termes d'attention cognitive de la part du patient, preuve que sa dynamique est satisfaisante. Il est donc envisageable que ce dispositif prenne place dans une consultation d'éducation planifiée, à distance d'un programme d'éducation thérapeutique ou dans le cadre d'un suivi éducatif ${ }^{[4]}$. Les modalités d'évaluation proposées dans ce dispositif peuvent surprendre car c'est le patient qui conduit la séance en choisissant les compétences à évaluer. De même, les critères d'évaluation sont partagés avec le patient et ce dernier est sollicité pour discuter les résultats obtenus et les décisions pédagogiques qui en découlent. Ainsi, par la négociation qu'il implique, ce dispositif d'évaluation pédagogique constitue un 
véritable outil de médiation entre le patient et le soignant. Ceci se confirme dans les réponses des patients qui estiment que le support leur a permis de mieux situer leurs apprentissages et surtout les partager avec leur entourage. Cette médiation vaut également entre les soignants qui voient un moyen de laisser une trace sur les transformations pédagogiques du patient et de partager les résultats avec le médecin traitant.

Cette centration sur le patient que propose le dispositif d'évaluation vient renforcer les pratiques actuelles d'ETP, au cours desquelles le patient doit être considéré comme un véritable acteur de sa prise en charge ${ }^{[16]}$. Cependant, pour mettre en œuvre le dispositif d'évaluation, un temps de préparation et d'appropriation reste nécessaire. C'est ainsi qu'une formation complémentaire devrait permettre aux soignants d'une part de travailler leurs représentations de l'évaluation et le sens qu'ils accordent à une évaluation à visée formative, et d'autre part de s'exercer à présenter le dispositif au patient et à mâ̂triser les techniques de questionnement adaptées à cette activité évaluative ${ }^{[25]}$.

\section{Limites de l'étude}

Il convient de rappeler que ce dispositif d'évaluation s'appuie sur une validité de contenu relative. Par ailleurs, l'étude de validité de construit proposée fait appel à des questionnaires dont seule la validité apparente peut être assurée. Il s'agit donc de résultats préliminaires. Cependant, malgré ces limites, ils ont permis d'identifier les insuffisances de ce dispositif d'évaluation, que d'autres études devraient venir confirmer.

Le pourcentage de patients étudiants (40\%) ayant participé à l'étude ne correspond pas vraiment au profil habituel des patients atteints de mucoviscidose. Ce résultat s'explique en partie par le fait que l'étude a été proposée à des patients de plus de 16 ans, les patients étudiants étant par ailleurs souvent plus disponibles que les adultes en activité professionnelle. Dans la mesure où l'objectif de l'étude ne s'intéresse pas à l'évolution des compétences mais à la perception d'utilité du dispositif d'évaluation, on peut considérer que les statuts de jeune adulte étudiant et jeune adulte professionnel, au regard de leur expérience de vie et de traitement, sont relativement semblables par rapport aux objectifs de l'étude et n'appellent pas une spécificité d'analyse du point de vue des réponses fournies.

\section{Conclusion}

Il ressort de cette étude que, dans le cadre de l'éducation thérapeutique du patient, le recours à un dispositif d'évaluation pédagogique est perçu comme utile. Le dispositif proposé vient alimenter les pratiques des soignants-éducateurs en leur permettant de renforcer la structuration des programmes d'ETP ${ }^{[26]}$ tel que le recommande la Haute Autorité de Santé (HAS). Il permet de structurer la rencontre évaluative en prenant en compte un ensemble de compétences et en permettant au patient d'être un véritable partenaire de son évaluation. Il offre les conditions d'une évaluation pédagogique valorisant les apprentissages réalisés par le patient et donnant respectivement au patient et au soignant la possibilité de comparer leurs propres jugements, afin de prendre des décisions consensuelles, adaptées aux réels besoins du patient. Ce dispositif nécessite une préparation pédagogique, de telle manière que les patients et les soignants s'en servent comme véritable outil de médiation éducative. Les résultats obtenus chez les patients interrogent les pratiques et programmes d'éducation actuels mis en place par les soignants. Ils les incitent à les améliorer, en particulier dans les séances dédiées au lien entre apprentissage de compétences d'autosoins et d'adaptation à la maladie. Enfin, ce dispositif d'évaluation montre qu'il est possible de rendre compte du développement de compétences des patients loin d'un contrôle médical où la notion de co-évaluation prend tout son sens. 


\section{Contributions}

Valérie David et Marythé Kerbrat ont coordonné la mise en ouvre de l'étude dans leur Centre de ressources et de compétences de la mucoviscidose $(\mathrm{CRCM})$ respectif. Jacqueline Iguenane a participé à la conception du protocole d'étude, a formé les soignants éducateurs impliqués dans l'étude, a recueilli et analysé les données et a participé à la rédaction de l'article. Claire Marchand a participé à la conception du protocole d'étude, au recueil et l'analyse des données et à la rédaction de l'article. Rémi Gagnayre a participé à la conception du protocole d'étude et à la rédaction de l'article.

Ce travail a fait l'objet d'une communication orale lors du congrès de la Société Française de Santé Publique, en septembre 2009 à Nantes.

\section{Remerciements}

Nous tenons à remercier les soignants éducateurs du CRCM de Roscoff (Dr Adirou, ML Madec et S. Pichon, M. Kerbrat) et du CRCM de Nantes (Dr V. David, C. Breville, P. Douaud), qui se sont investis dans cette étude ainsi que les patients atteints de mucoviscidose ayant accepté de participer à l'étude.

\section{Références}

1. HAS-INPES. Structuration d'un programme d'éducation thérapeutique du patient dans le champ des maladies chroniques. Guide méthodologique. 2007 [On-line] Disponible sur : http://www.has-sante.fr/ portail/upload/docs/application/pdf/etp_-_ guide_version_finale_2_pdf.pdf.

2. Ivernois (d') JF, Gagnayre R. Éduquer le patient. Paris : Vigot, 2008.

3. Ivernois (d') JF, Gagnayre R. Mettre en œuvre l'éducation thérapeutique. Actualité et dossier en santé publique 2001;36:11-3.

4. HAS. Éducation thérapeutique du patient. Définition, finalités et organisation. Recommandations. 2007 [On-line] Disponible sur : http://www.has-sante. $\mathrm{fr} /$ portail/plugins/ModuleXitiKLEE/types/ FileDocument/doXiti.jsp?id=c_604959.
5. Ivernois (d') JF, Gagnayre R, Assal JP, Golay A, Deccache A, Libion F. Propositions pour l'évaluation de l'éducation thérapeutique du patient. Actualité et dossier en santé publique 2007;58:49-54.

6. Lerbet G. Système, personne et pédagogie. Paris : ESF ( $2^{\mathrm{e}}$ ed.), 1993.

7. Gagnayre R, Marchand C, Pinosa C, Brun MF, Billot D, Iguenane J. Approche conceptuelle d'un dispositif d'évaluation pédagogique du patient. Pédagogie Médicale 2006;7:31-42.

8. Joannert P. Compétences et socioconstructivisme : un cadre théorique. Bruxelles : de Boeck Universités, 2002.

9. INED Vaincre la Mucoviscidose, Observatoire National de la Mucoviscidose, rapport sur la situation de la Mucoviscidose, 2002-2003, Paris, 2005.

10. Circulaire DHOS/O 1/DGS/SD 5 n 2001-502 du 22 octobre 2001 du 22 octobre 2001 relative à l'organisation des soins pour la prise en charge des patients atteints de mucoviscidose.

11. David V, Iguenane J, Ravilly S. L'éducation thérapeutique dans la mucoviscidose : quelles compétences pour le patient ? Rev Mal Respir 2007;24:57-62.

12. David V, Iguenane J, Greffier C, Gagnayre R, Ravilly S. Le conducteur pédagogique : une aide pour mener des séances d'éducation thérapeutique. L'exemple du «planning du souffle » dans la mucoviscidose. Rev Mal Respir 2008;25:1322-5.

13. Scallon G. L'évaluation des apprentissages dans une approche par compétences, Bruxelles : De Boeck, 2004.

14. Legendre R. Dictionnaire actuel de l'éducation. Montréal : Édition Guérin ( $3^{\mathrm{e}}$ éd), 2005.

15. Develay M. Propos sur les sciences de l'éducation. Paris : ESF, 2001.

16. OMS Bureau Régional pour l'Europe, Éducation Thérapeutique du Patient, programmes de formation continue pour professionnels de soins dans le domaine de la prévention des maladies chroniques, Copenhague 1998.

17. Poussin G. La pratique de l'entretien clinique, Paris : Dunod, 1994.

18. François Ph. Sentiment d'efficacité et compétences : une approche sociale cognitive. Éducation permanente 1998;135:45-55.

19. Barrier $\mathrm{Ph}$. L'autonormativité du patient chronique : un concept novateur pour la relation de soin et l'éducation thérapeutique, ALTER. European Journal of Disability Research 2008;2:271-91.

20. Noël B. La métacognition, Bruxelles : De Boeck Université ( $2^{\mathrm{e}}$ éd), 1997. 
21. Carré P, Moisan A, Poisson D. L'autoformation. Psychopédagogie, Ingénierie, Sociologie. Paris : PUF (2 éd.), 2002.

22. Bandura A. «J'y arriverai » : le sentiment d'efficacité personnelle. Paris : Sciences Humaines 2004;148:42-5.

23. Doron R, Parot F. Dictionnaire de Psychologie. Paris : PUF, 1998.

24. Pellechia A, Gagnayre R. Entre cognition et émotion : les potentialités de l'art dans l'éducation thérapeutique. Pédagogie Médicale 2006;7:101-9.
25. Vermersch P. L'entretien d'explicitation en formation initiale et continue. Paris : ESF, 1994.

26. Ivernois (d') JF, Gagnayre R. Les programmes structurés d'éducation thérapeutique. Actualité et dossier en santé publique 2009;66:33-4.

Correspondance et offprints : Claire Marchand, Laboratoire de Pédagogie de la Santé, UFR-SMBH, 74 rue Marcel Cachin ; 93017 Bobigny Cedex, France.

Mailto : c.marchand@smbh.univ-paris13.fr 